\title{
AN INTELLECTUAL TRAFFIC ORGANISM FOR VANET
}

\author{
R. Dhaya ${ }^{1}$, R. Amirthavalli ${ }^{2}$, N. Raghavi ${ }^{3}$, K. Elakkiya ${ }^{4}$, \& B. Kamali $^{5}$
}

\begin{abstract}
Absract- As of now, the emergency services provided for accidents are not very effective as it is based on the imprecise or insufficientdata gathered from the accident spot. Any assessment which provides quality information on accident will help in the victims' rescue. To collect such quality information Vehicular Adhoc Networks(VANET) can be deployed, which can assess the seriousness of the accident. This paper proposes an estimation technique which uses classification algorithm in data mining. To train the classifier the previous accident historical data can be used. Thus this technique focuses on deploying effective emergency services based on the accurate data collected by VANET.
\end{abstract}

Keywords : Automatic accident system, Severity Estimation, Traffic control system, Intelligent Accident control system.

\section{INTRODUCTION}

There is a steady increase in number of vehicles on roads these days, which has effected in increasein number of fatal accidents on roads. Steps should be taken to mitigate the number of deaths due to accident as this poses a grave problem to the society. Vehicular Ad hoc Networks (VANET), a part of Intelligent Transport Systems(ITS), can be deployed in future vehicles to mitigate the number of deaths due to accidents. In VANETs, the data is communicated between either vehicle to vehicle(V2V) directly or vehicle to road side infrastructure(V2I) [2].

Safety can be enhanced by deploying sensors capable of both sensing and communicating with other vehicles and infrastructures used for communication. Thus with these kinds of intelligent vehicles, the victims, in case of an accident, can be rescued and treated appropriately and thus can decrease the mortality rate. These systems must be intelligent enough to assess the seriousness of the accident as well present the course of actions needed to rescue as well save the life of the victims.

\section{ARCHITECTURE OVERVIEW}

VANET architecture, Figure1, in general consists of On-Board unit(OBU) in vehicles, a Control Unit(CU) and Road Side Units(RSU) which should be available on roads. Vehicles should be integrated with the OBU. The OBU uses sensors to sense any accident and also collect the information regarding the severity of the collision in case of accidents. The information about accident and all the data collected during accident is relayed to the CU via V2V or V2I. CU is responsible for handling the accident situation further by alerting appropriate emergency services required for rescuing the victim.

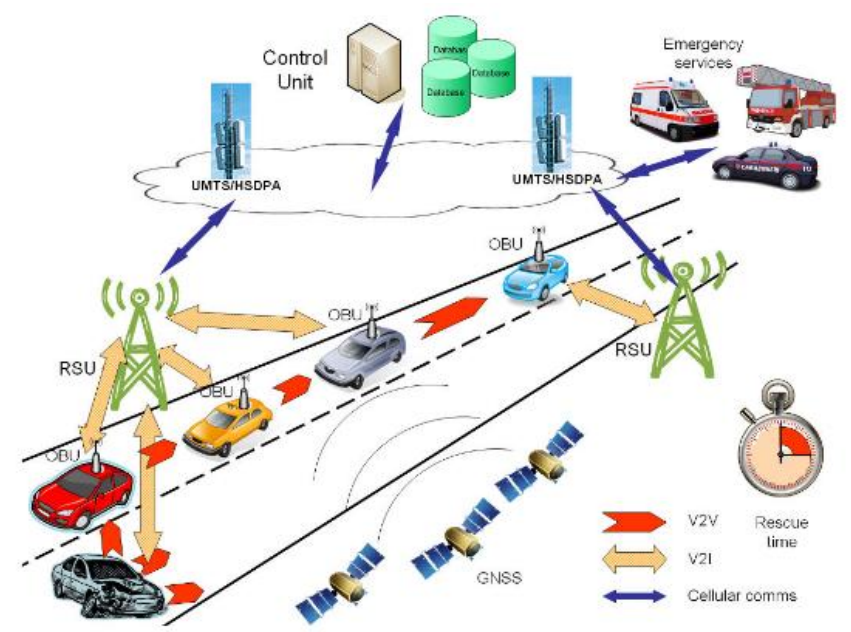

Figure. 1. Architecture of automatic accident notification and assistance using vehicular networks

\footnotetext{
${ }^{1}$ Department of Computer Science and Engineering, Rajalakshmi Engineering College, Chennai.

${ }^{2}$ Department of Computer Science and Engineering, Velammal Engineering College, Chennai.

${ }^{3}$ Department of Computer Science and Engineering, Velammal Engineering College, Chennai.

${ }^{4}$ Department of Computer Science and Engineering, Rajalakshmi Institute Of Technology, Chennai.

${ }^{5}$ Department of Computer Science and Engineering, Rajalakshmi Institute Of Technology, Chennai.
} 
The OBU should be designed in such a way that it must be feasible to deploy in low cost as well as it must be open for future software updates The hardware design of OBU that is to be integrated in the vehicles can initially be of specific purpose but in long run the design must be of general purpose so that new services can be included in future [5].

The OBUs relay the accident information to CU via internet which can be through RSUs or through vehicles that is acting as Internet gateways. If there is no direct communication between the vehicle and $\mathrm{Cu}$, then the same information can be broadcast to the vehicles on the road which serves two purpose, i) notifies all vehicles moving on the same road about the accident and ii) the information is also relayed to $\mathrm{CU}$ through $\mathrm{V} 2 \mathrm{~V}$ communication $[6,7]$.

This architecture provides communication of the vehicles involved in accident, assessment of the impact of the accident to the CU. Based on the data collected, $\mathrm{CU}$ will alert the emergency services required to speed up the rescue operations.

\subsection{Structureof On-Board Unit}

The responsibility of OBU is to detect the accident and collect the information regarding accident from sensors and transmit all these data to $\mathrm{CU}$ directly, or through other vehicles. Also to notify the other vehicles about such an accident to avoid traffic and as well as other undesirable events.

The OBU system in Figure 2,shows the interaction between sensors, the data acquisition unit, the processing unit, and wireless interfaces.

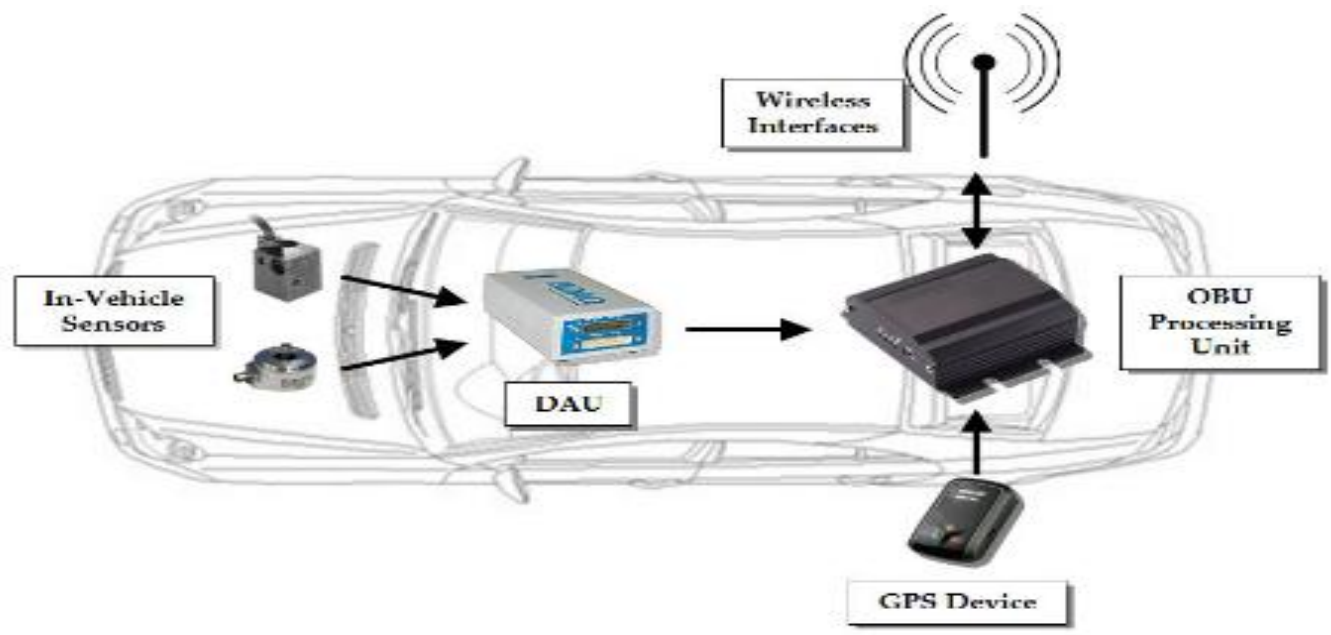

Fig. 2.Structure of On-Board Unit

Data Acquisition Unit (DAU) collects the data from the sensors in the vehicle at regular intervals, converts into a common format. This information is sent to the OBU Processing Unit.

\subsection{Structure Ofcontrol Unit}

The control Unit $(\mathrm{CU})$ receives the warning message from the OBU of a vehicle which met with accident and also information about the severity of the accident. Based on the warnings, the $\mathrm{CU}$ alerts the corresponding emergency services for quick recovery of victims at the accident spot.

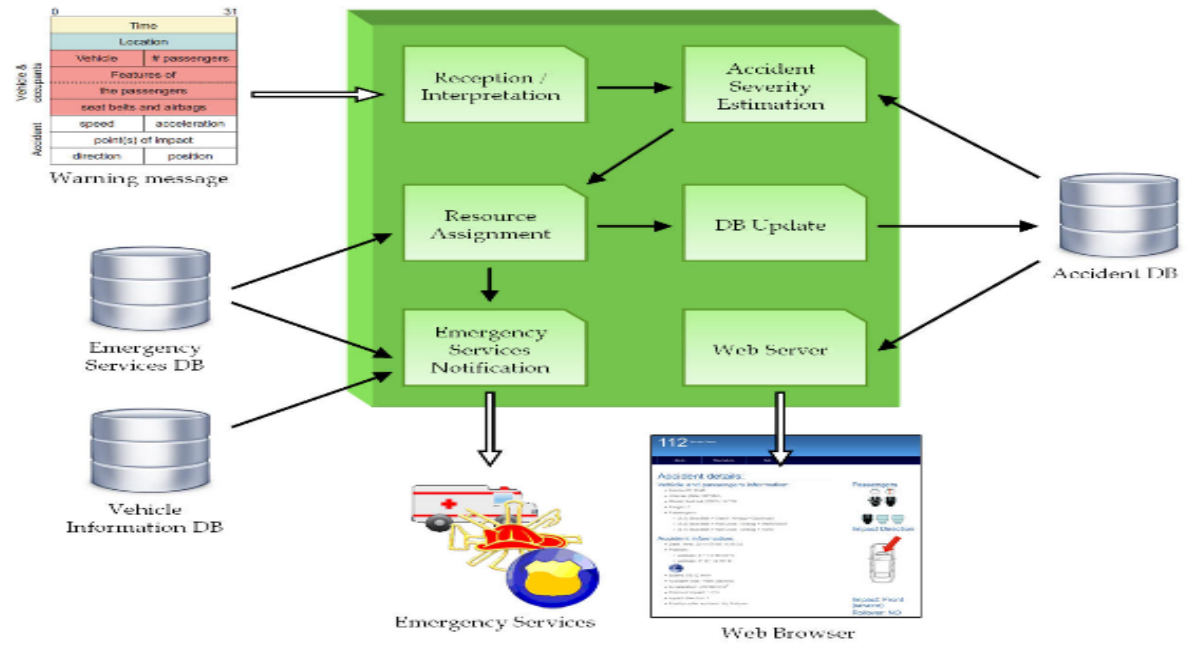

Fig. 3.Structure of Control Unit. 
The scope of this paper is two folds. One, deals with vehicle damage and the other collects information about the impact of accident on the victims in the vehicle. The assessment of the damage can help in judging the victims' status and further course if actions. Additionally, the knowledge from the previous accident databases also can refine the course of actions, thus improving the rescue process.

Steps taken in KDD and its results are dicussed in the following sections.

\subsubsection{Data Acquisition, Selection And Preprocessing Steps}

To assess any accident the previous history of accidents' data set is needed for algorithm development. So relevant data sets has to be identified with the following attributes : Accident, Vehicle, Victims, which contains details about the characteristics of both the crash and environmental conditions, vehicles and victims involved while the accident happened.

The data thus identified has to be preprocessed so that noisy, incomplete, inaccurate data are removed from the data set and contains only valid data.

\subsubsection{Transformation Step}

This step takes care of finding the relevant features in the selected data set by reduction and projectionthat will be used for KDD process. Some of the variables that could be used like speed of the vehicle, status of air bag during accidents are collected from the OBU of the vehicle, also can help fill up the data for the future data sets in this system.

Obtaining victims' individual characteristics is not feasible. But some characteristics like sex, age can be obtained to some extent which can improve the system prediction accuracy $[9,10]$.

\section{MODULE DESCRIPTION}

\subsection{Reception/Interpretation Module}

In $\mathrm{CU}$, the reception module waits for any kind of notification from OBU of any vehicle, in case of accidents. Apart from notification, it also collects other information like the type of the vehicle, the route, speed and the type of accident (front, back etc).

This information is sent to assessment module for further processing.

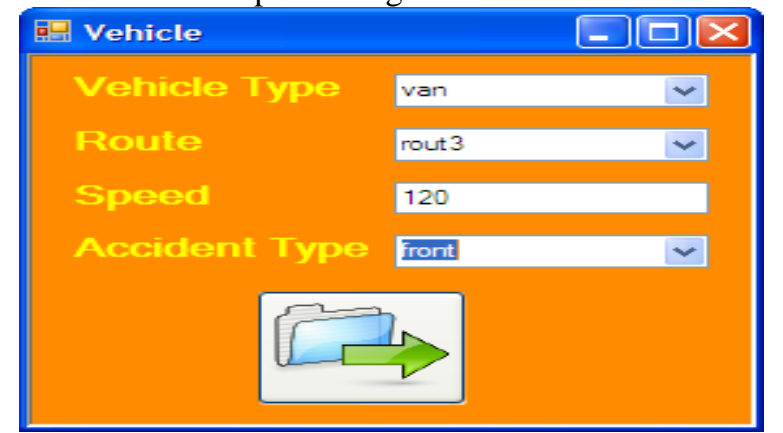

Figure 4: Reception/Interpretation module collecting data

The vehicle automatically gets the values of vehicle type route, speed and accident type successfully

\section{2 .Accident Severity Assessment Module}

This module will assess the causalities of the victims whenever a notification of any accident is received. Based on the assessment the Resource Assignment module defines the resources need to handle the recovery.

\subsection{Database Update Module}

The accident database which stores all the data about accidents is updated by this module whenever the CU receives a new accident notification, thereby expanding the data set for future reference.

\subsection{Web Server Module}

A Web server is part of the CU which is used for viewing the accident history and also the current one to estimate the requirement of assistance. It also provides a web interface for the users. 


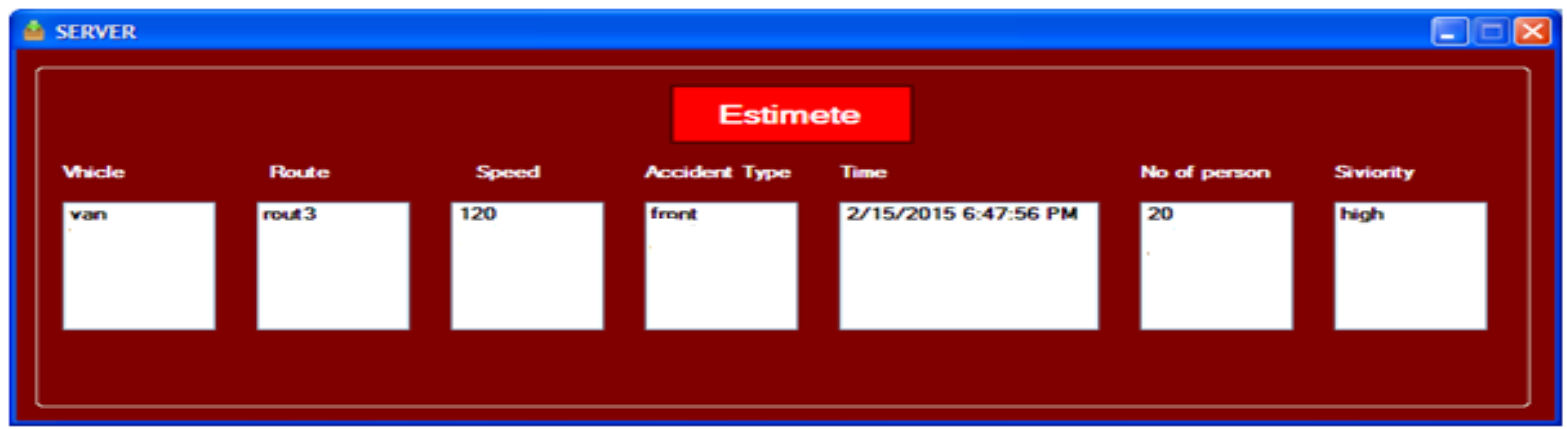

Server estimates the number of person and severity successfully by passing the unit testing

\subsection{Emergency Services Notification Module}

The Resource Assignment module after defining necessary resources required for recovery operation, alerts the appropriate emergency services so as to initiate the rescue task. The following data are also sent to these services: i) severity of the accident on both the vehicle as well the victims' involved ii) the resource set defined iii) standard rescue procedure for the vehicles involved.

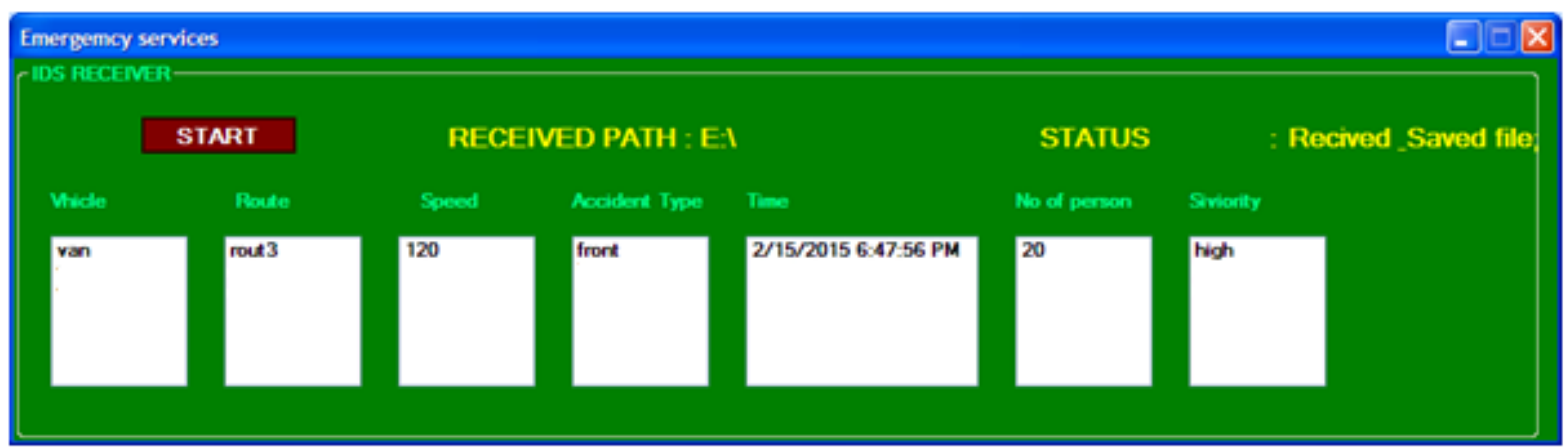

Web server alert is activated efficiently and the solution is explored or generated

\subsection{Transformation Phase}

This step takes care of finding the relevant features in the selected data set by reduction and projection that will be used for KDD process.

\subsection{Data Mining And Interpretation/Evaluation Phases}

Classification, a data mining technique is used for identifying the class to which the current accident belong to. The previous history of accident database is used as data set which identifies the current accident's class. With the help of this the kind of rescue operations and the resources needed to carry out the rescue operations are identified.

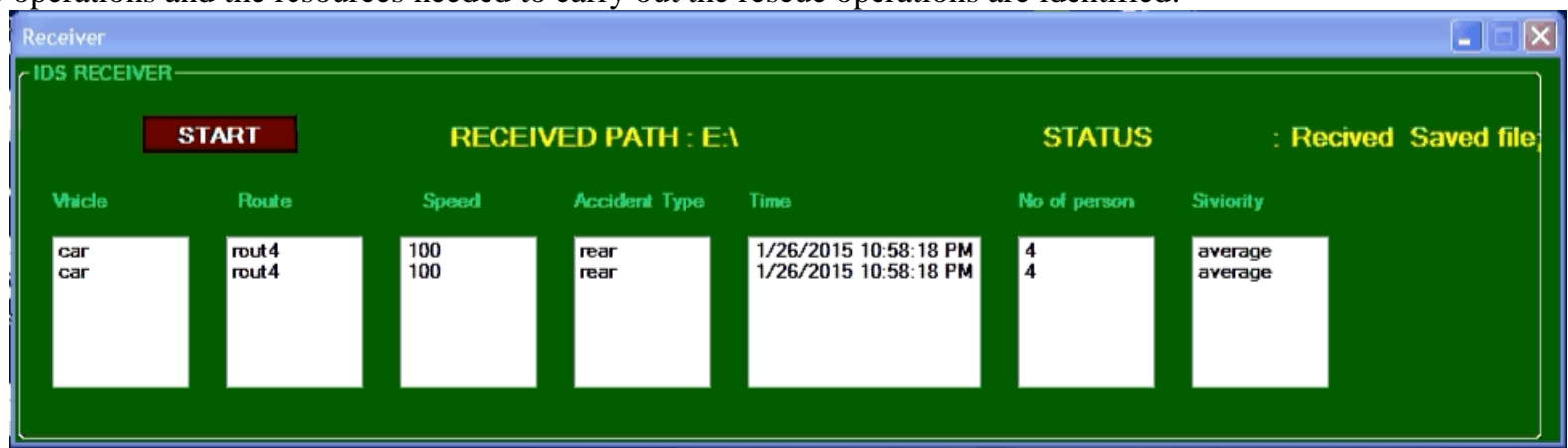

Server sends the data with severity to web server at the same time it stores the data in database history table by achieving simultaneous integration with database and web server

\section{CONCLUSION AND FUTURE SCOPE}

This paper intended to improve the time taken to initiate the rescue operation during accidents as well provide the information about the accident which may provide an improved, efficient rescue operation. To achieve this, VANET, an Intelligent Transport System (ITS), is deployed to notify and convey the accident and the information about the accident to a Control Unit which initiates the emergency services and other resources required for rescue operation without the need of human intervention. 
The future work can focus on the classification algorithm further to increase the system accuracy. The system developed is a prototype which can be developed to include warning messages to the friends and relatives of the victim and also convey the rescue operations that is being carried out and the where the victim is taken to further treatment as future work.

\section{REFERENCES}

[1] Dirección General de Tráfico (DGT), "The main statisticsof road accidents. Spain," 2010,

[2] Eurostat: Statistical Office of the European Communities, "Transport statistics in the EU," 2012.

[3] J. Miller, "Vehicle-to-vehicle-to-infrastructure (V2V2I) intelligent transportation system architecture," in IEEEIntelligent Vehicles Symposium, june 2008, pp. 715-720.

[4] F. Martinez, C.-K.Toh, J.-C. Cano, C. Calafate, and P. Manzoni, "Emergency services in future intelligent transportationsystems based on vehicular communication networks,"IEEE Intelligent Transportation Systems Magazine, vol. 2, no. 2,pp. 6-20, summer 2010.

[5] M. Fogue, P. Garrido, F. J. Martinez, J.-C.Cano, C. Calafate, and P. Manzoni, "Prototyping an automatic notificationscheme for traffic accidents in vehicular networks," in4th IFIP Wireless Days Conference, October 2011.

[6] M. Fogue, P. Garrido, F. J. Martinez, J.-C. Cano, C. T. Calafate, and P. Manzoni, "Evaluating the impact of a novelmessage dissemination scheme for vehicular networks usingreal maps,”Transportation Research Part C: EmergingTechnologies, vol. 25, pp. 61-80, December 2012.

[7] M. Hall, E. Frank, G. Holmes, B. Pfahringer, P. Reutemann, and I. H. Witten, “The WEKA data mining software: anupdate,"SIGKDD Explorations, vol. 11, pp. 10-18, November 2009.

[8] National Highway Traffic Safety Administration (NHTSA), "NationalAutomotiveSamplingSystem (NASS) andGeneral EstimatesSystem (GES)," 2012.

[9] K. Yu and E. Dutkiewicz, "Geometry and Motion-Based Positioning Algorithms for Mobile Tracking in NLOSEnvironments,"Mobile Computing, IEEE Transactions on, vol. 11, no. 2, pp. 254-263, feb. 2012.

[10] M. Hall, “Correlation-based feature selection for machine learning," Ph.D. dissertation, Department of ComputerScience, University of Waikato, Hamilton, New Zealand, 2008. 